\title{
ene
}

\section{DIFERENCIAS DE CARGA EN EL CUIDADO ASOCIADAS AL GÉNERO}

\section{DIFFERENCES IN BURDEN OF CARE ASSOCIATED WITH GENDER}

\author{
Carmen María Fernández Torralbo (1), Irene Cueto Torres (2), Maria Luisa \\ Grande Gascón (3)
}

(1) Hospital El Escorial, España

(2) Hospital Clínico Universitario Lozano Blesa, España

(3) Universidad de Jaén, España

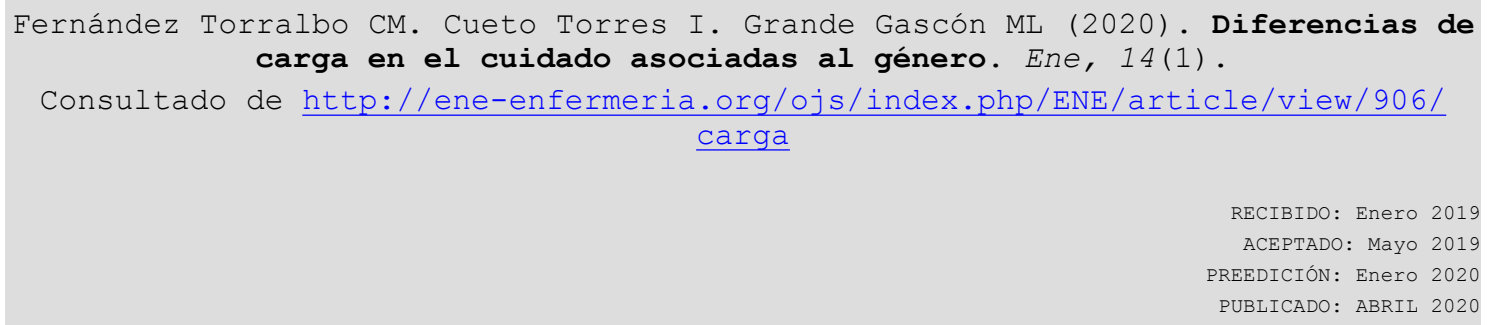




\section{Resumen}

El cuidado informal es un sistema de cuidados que sostienen una sociedad cada vez más envejecida y dependiente.

Se sabe que es llevado a cabo en su mayoría por mujeres, pero no se había estudiado si estadísticamente éstas sufren más carga asociada al cuidado que los hombres. Nuestro objetivo es estudiar la diferencia en la carga subjetiva asociada al cuidado. Para ello realizamos una búsqueda en bases de datos bibliográficas de artículos que estudiaban la carga asociada al género, de la cual obtuvimos cinco artículos. Como resultados obtuvimos que no existe solamente un mayor número de mujeres que cuidan, sino que estas experimentan mayor carga a todos los niveles como son la carga psicológica, tensión asociada al cuidado y dificultades para llevar a cabo la tarea del cuidado, síntomas depresivos y cambios negativos en su vida a causa del cuidado. Queda mucho camino en la investigación, pero la base es que los y las profesionales de salud conozcamos nuestra importancia en abordar los problemas con perspectiva de género.

Palabras clave: género y salud; atención de enfermería; enfermería de atención primaria;

\section{Abstract}

Informal care is a system of care that supports an increasingly aging and dependent society. It is known that it is carried out mostly by women, but it had not been studied if statistically these suffer more burden associated with care than men. Our objective is to study the difference in the subjective load associated with care. To do this, we carried out a search in bibliographic databases of articles that studied the burden associated with gender, from which we obtained five articles. As results we obtained that there is not only a greater number of women who care, but that they experience greater burden at all levels such as the psychological burden, tension associated with care and difficulties to carry out the care task, depressive symptoms and changes negative in your life because of care. There is still a long way to go in research, but the basis is that health professionals know our importance in addressing problems with a gender perspective.

Keywords: gender and health; nursing care; primary care nursing; 


\section{INTRODUCCIÓN}

A la hora de hablar de cuidado informal, hablamos de una gran red de cuidados llevados a cabo de manera invisible, que soportan el sistema de Estado y sociedad en el que vivimos. (1)

Quien lleva a cabo estos cuidados son normalmente hijas, esposas 0 madres de la persona cuidada. (2) Concretamente, el perfil de persona cuidadora se corresponde con una mujer de 60 años de media, ama de casa y con un nivel de estudios bajo.(3) Las mujeres suponen el $84 \%$ de las cuidadoras, lo que nos hace plantearnos si el sesgo de género afecta a este cuidado, ya no sólo en la forma en que se reparte sino en la forma en que se vive esta carga.(4)

Cuando hablamos de carga asociada al cuidado, hablamos del trabajo y esfuerzo adicional necesario para satisfacer las necesidades de las personas cuidadas.5) Este concepto se compone de muchos otros como son el estrés, los sentimientos negativos asociados al cuidado, las dificultades relacionadas con el cuidado y por supuesto aspectos de salud física, psíquica, sociales y financieros.(6)

Esta carga asociada al cuidado aumenta conforme aumenta la población que necesita de estos cuidados. Los continuos avances en salud hacen que cada vez vivamos más tiempo, haciendo que la población envejezca. La pirámide de población está cambiando hacia una sociedad más envejecida, formada por individuos con cada vez más enfermedades crónicas, mayores dificultades para el autocuidado y cuyos cuidados son llevados a cabo por las ya mencionadas cuidadoras informales.(6)

\section{Objetivo}

Analizar la diferencia en la carga subjetiva asociada al cuidado, teniendo en cuenta el sesgo de género que existe entre quienes llevan a cabo el cuidado informal.

En cuanto a los objetivos específicos distinguimos:

- Estudiar el estigma asociado al cuidado

- Comparar la carga social asociada al cuidado

- Identificar si existen cambios vitales asociados al cuidado de forma predominante en alguno de los géneros

- Comprobar si la tensión asociada al cuidado es mayor en un género $u$ otro

- Analizar si existe diferencia entre géneros asociada a los síntomas depresivos 


\section{MATERIAL Y MÉTOdOS}

Llevamos a cabo una búsqueda en bases de datos bibliográficas en las bases de datos Cochrane, Cuiden plus, Lilacs, Cinhal, CSIC, Proquest, Medline y Pubmed con las palabras clave "mujer y cuidadora" y en inglés "women and caregiver". Llevamos a cabo una búsqueda inicial, con un amplio margen de resultados. Es por esto que decidimos acotar los resultados a aquellos que compartieran ciertos criterios:

- Que los artículos tuvieran una antigüedad máxima de 10 años.

- Que estuvieran disponibles a texto completo de forma gratuita.

- Que los textos estuvieran escritos en español o inglés

- Que analizaran la relación existente entre la forma de cuidar y el género.

Tras esto, obtuvimos 401 resultados. Todos ellos fueron leídos a texto completo, tras lo cual nos quedamos con 36 artículos. Decidimos acotar a aquellos con los que pudiéramos llevar a cabo una revisión y comparación sistemática acerca de las diferencias en la carga subjetiva del cuidado. Finalmente, obtuvimos cinco resultados. 


\section{REsultados}

\section{Características de los estudios}

\begin{tabular}{|c|c|c|c|c|}
\hline AUTOR Y AÑO & $\begin{array}{l}\text { NÚMERO DE } \\
\text { PARTICIPANTES }\end{array}$ & $\begin{array}{l}\text { TIPO DE } \\
\text { ESTUDIO }\end{array}$ & $\begin{array}{l}\text { CARACTERÍSTICAS } \\
\text { DE LA POBLACIÓN }\end{array}$ & RESULTADOS \\
\hline $\begin{array}{l}\text { Perrin et al., } \\
2015^{7}\end{array}$ & 81 & $\begin{array}{l}\text { Descriptivo } \\
\text { transversal }\end{array}$ & $\begin{array}{l}\text { Cuidadores de } \\
\text { pacientes con } \\
\text { escle ros is } \\
\text { múltiple }\end{array}$ & Carga psicológica \\
\hline $\begin{array}{l}\text { K h a n } \\
\text { Wishart, } \\
\text { Randolph y } \\
\text { Santulli, } \\
2016^{8}\end{array}$ & 82 & $\begin{array}{l}\text { Descriptivo } \\
\text { transversal }\end{array}$ & $\begin{array}{l}\text { Cuidadores de } \\
\text { pacientes con } \\
\text { demencia }\end{array}$ & Carga asociada al cuidado \\
\hline $\begin{array}{l}\text { A kp ın a r, } \\
\text { Küçügüçiü } \\
\text { y Ye n e r, } \\
2011^{9}\end{array}$ & 192 & $\begin{array}{l}\text { Descriptivo } \\
\text { transversal }\end{array}$ & $\begin{array}{l}\text { Cuidadores de } \\
\text { pacientes con } \\
\text { alzheimer }\end{array}$ & Carga asociada al cuidado \\
\hline $\begin{array}{l}\text { B r a z i l, } \\
\text { Thabane, } \\
\text { Foster y } \\
\text { B é d r a d, } \\
2009^{10}\end{array}$ & 283 & $\begin{array}{l}\text { Descriptivo } \\
\text { transversal }\end{array}$ & $\begin{array}{l}\text { Cuid a d o res } \\
\text { informales de su } \\
\text { pareja terminal }\end{array}$ & Tensión asociada al cuidado \\
\hline $\begin{array}{l}\text { Je s s u } \\
\text { B a k a } \mathrm{s}, \\
\text { McLennon } \\
\text { y Weaver, } \\
2015^{11}\end{array}$ & 243 & $\begin{array}{l}\text { Descriptivo } \\
\text { transversal }\end{array}$ & $\begin{array}{l}\text { Cuidadores de } \\
\text { supervivientes } \\
\text { de ictus }\end{array}$ & $\begin{array}{l}\text { Dificultades para llevar a cabo la tarea del } \\
\text { cuidado, síntomas depresivos y cambios } \\
\text { negativos en su vida a causa del cuidado. }\end{array}$ \\
\hline
\end{tabular}

2. Descripción de los resultados de la revisión

En este apartado incluiremos los artículos de Khan, Wishart, Randolph y
Santulli por un lado y Akpınar, Küçügüçiü y Yener. Ambos son comparables ya que estudian la carga asociada al cuidado, pero además ambos reflejan la realidad del cuidado a pacientes de salud mental.

\begin{tabular}{|c|c|c|c|c|}
\hline ESTUDIO & MUESTRA & $\begin{array}{l}\text { INSTRUMENTOS } \\
\text { DE MEDICIÓN } \\
\text { UTILIZADOS }\end{array}$ & PROCEDIMIENTO & FACTORES MEDIDOS \\
\hline $\begin{array}{l}\text { A k p ın a r, } \\
\text { Küçügüçiü y } \\
\text { Yener, } 2011\end{array}$ & $\begin{array}{l}120 \text { mujeres y } 72 \\
\text { h o m b r e s } \\
\text { cuidadores de } \\
\text { personas con } \\
\text { alzheimer }\end{array}$ & $\begin{array}{l}\text { - Cuestionario de } \\
\text { característic as } \\
\text { sociodemográficas. } \\
\text { - Mini-mental State } \\
\text { examination. } \\
\text { - I v e n t r i o } \\
\text { neuropsiquiátrico de } \\
\text { Cummings. } \\
\text { Caregiver burden } \\
\text { inventory. }\end{array}$ & $\begin{array}{l}\text { En dos hospitales con } \\
\text { unidades para pacientes con } \\
\text { demencia se informó a los } \\
\text { pacientes sobre la mecánica } \\
\text { del estudio y se pidió su } \\
\text { consentimiento, tras lo cual } \\
\text { rellenaron los cuestionarios. }\end{array}$ & $\begin{array}{l}\text { - Nivel de educación. } \\
\text { - } \text { Ingresos en relación a los gastos. } \\
\text { - } \text { Prabajo. } \\
\text { - } \text { Reriodo de cuidado. } \\
\text { - Edad del con la persona cuidador } \\
\text { - } \text { Horas diarias dedicadas al cuidado } \\
\text { - } \text { Género del cuidador } \\
\text { - } \text { Estado de la persona cuidada } \\
\text { - Carga asociada al cuidado }\end{array}$ \\
\hline
\end{tabular}


FERNÁNDEZ-TORRALBO - CUETO-TORRES - GRANDE-GASCóN DIFERENCIAS DE CARGA EN EL CUIDADO ASOCIADAS AL GÉNERO

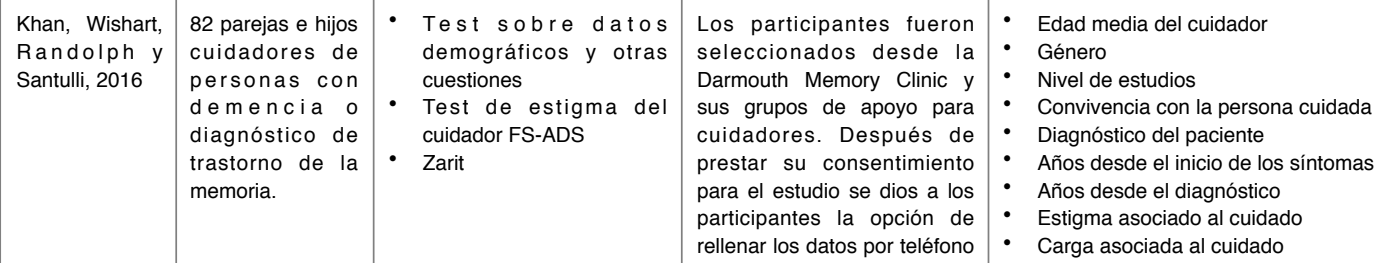

En el primer estudio de Akpınar, Küçügüçiü y Yener, como podemos observar la muestra fue de 192 personas en total. Se consiguió mediante entrevista y cuestionario en los dos hospitales de Turquía que disponían de las mejores unidades de demencia. Para ello se utilizaron los siguientes instrumentos:

Cuestionario de características sociodemográficas. Fue preparado por los investigadores para disponer de una base sociodemográfica que sirviera para la comparación de los diferentes datos sociodemográficos.

Mini-mental State examination (MMSE). Es una herramienta de consenso internacional para la evaluación de la demencia. Es una prueba escrita con una puntuación máxima de 30 , en la que las puntuaciones inferiores indican problemas cognitivos. (12)

Inventario neuropsiquiátrico de Cummings (NPI): Mide la frecuencia y gravedad de 12 signos y síntomas clínicos como son la desilusión, alucinaciones, agitación, depresión, ansiedad, euforia, apatía, desinhibición, labilidad, nivel de actividad motora, cambios del ciclo del sueño y desórdenes alimentarios.
El rango de puntuación se encuentra entre 1 y 144, existiendo mayor deterioro cuanto mayor sea la puntuación. (9)

Caregiver burden inventory (CBI). Es una herramienta autoadministrada de 24 ítems que mide el grado de carga asociada al cuidado que perciben los cuidadores. (9)

Los resultados se midieron mediante t de Student para variables cuantitativas (edad media del cuidador, tiempo medio dedicado al cuidado, edad media de los pacientes, MMSE y NPI) y chi cuadrado para variables cualitativas 0 mixtas (nivel de educación, ingresos mensuales, situación laboral y duración del cuidado). Un intervalo de confianza del 95\% (es decir, una $\mathrm{p}<0.05$ ) se consideró estadísticamente significativo.

La carga asociada al cuidado fue mayor en mujeres que en hombres $(p=0.002)$. Analizando los componentes que intervienen en esta carga, se encontró que todos ellos eran mayores en el caso de las mujeres que en el de los hombres: tiempo de cuidado $(p=0.04)$, carga física $(p=0.00)$ y carga social $(p=0.04)$. 
En el segundo estudio, de Khan, Wishart, Randolph y Santulli, se contó con una muestra de 82 personas. Mediante e-mail o teléfono se completaron los siguientes instrumentos:

Test sobre datos demográficos y otras cuestiones: desarrollado por los autores, mide factores como la edad, el género, el nivel de estudios, etc.

Test FS-ADS modificado: consistente en 18 ítems que se responden mediante una escala Likert del 1 al 5 , donde 1 significa el nivel más bajo y el 5 el más alto. Escalas más altas se asocian con un nivel más alto de estigma. Las preguntas fueron adaptadas y modificadas en función de si se administraba a una pareja o descendiente de la persona cuidada. (8)

Escala de Zarit: mide la carga de cuidado. Se completa de igual forma mediante una escala Likert del 0 al 4 , donde 0 significa el nivel más bajo y el 5 el nivel más alto. Escalas más altas se asocian con mayor carga asociada al cuidado. (8)

En cuanto a los resultados, podemos resaltar en primer lugar que existe una relación directa positiva entre estigma y carga, aumentando la una conforme aumenta la otra. Esta relación fue igual para todos los grupos de edad y géneros $(p<0.05)$. En cuanto a la relación de cada una de ellas con el género de la persona cuidadora, las mujeres cuidadoras experimentan más estigma asociado al cuidado que los hombres $(p<0.001)$ y mayor carga $(p=0.009)$.

En relación al primer estudio de Akpınar, Küçügüçiü y Yener, podemos sacar como conclusión que la carga asociada al cuidado fue mayor en mujeres que en hombres $(p=0.002)$. Analizando los componentes que intervienen en esta carga, se encontró que todos ellos eran mayores en el caso de las mujeres que en el de los hombres: tiempo de cuidado $(p=0.04)$, carga física $(p=0.00)$ y carga social $(p=0.04)$.

En cuanto a los resultados del segundo estudio, de Khan, Wishart, Randolph y Santulli, podemos resaltar en primer lugar que existe una relación directa positiva entre estigma y carga, aumentando la una conforme aumenta la otra. Esta relación fue igual para todos los grupos de edad y géneros. En cuanto a la relación de cada una de ellas con el género de la persona cuidadora, las mujeres cuidadoras experimentan más estigma asociado al cuidado que los hombres $(p=0.001)$ y mayor carga $(p=0.009)$.

Podemos concluir pues, en base a los resultados de ambos artículos, que muestran de forma coherente una relación estadísticamente significativa entre el hecho de ser mujer y cuidadora y experimentar carga asociada al cuidado. 
Los siguientes estudios, si bien muestran el mismo problema, lo hacen desde un abordaje menos específico, estudiando los factores en pacientes variados.

\begin{tabular}{|c|c|c|c|c|}
\hline ESTUDIO & MUESTRA & $\begin{array}{l}\text { INSTRUMENTOS DE } \\
\text { MEDICIÓN } \\
\text { UTILIZADOS }\end{array}$ & PROCEDIMIENTO & FACTORES MEDIDOS \\
\hline $\begin{array}{l}\text { Perrin et al., } \\
2015\end{array}$ & $\begin{array}{l}81 \text { cuidadores de } \\
\text { personas con } \\
\text { esclerosis múltiple }\end{array}$ & $\begin{array}{l}\text { - Información demográfica } \\
\text { - Cuestionario sobre la salud } \\
\text { del paciente-9 (PHQ-9) } \\
\text { - Escala de satisfacción con la } \\
\text { vida (SWLS) } \\
\text { - Escala de autoestima de } \\
\text { Rosemberg (RSES) } \\
\text { - Inventario de an siedad } \\
\text { - Estado-Rasgo (STAI) } \\
\text { - Inestionario de Zarit } \\
\text { Evaluation List (versión corta) } \\
\text { (ISEL-12) } \\
\text { - Cuestionario de salud (versión } \\
\text { corta) (SF-36) }\end{array}$ & $\begin{array}{l}\text { Se seleccionó la } \\
\text { muestra de la } \\
\text { Fundación Mexicana de } \\
\text { Esclerosis Múltiple y el } \\
\text { Departamento de } \\
\text { Neurociencia de la } \\
\text { Universidad de } \\
\text { Guadalajara (México). } \\
\text { Se recogieron los datos } \\
\text { en una entrevista de } 40 \\
\text { minutos. }\end{array}$ & $\begin{array}{l}\text { - Género } \\
\text { - Satisfacción con la vida } \\
\text { - } \text { Tepresión } \\
\text { - Apoyón social } \\
\text { - Autoestima } \\
\text { - Ansiedad } \\
\text { - Función física } \\
\text { - Runción psíquica } \\
\text { - } \text { Vol social } \\
\text { - Bienestad emocional } \\
\text { - Funcionamiento social } \\
\text { - Dolor } \\
\text { - Salud en general }\end{array}$ \\
\hline $\begin{array}{l}\text { B r a z i l, } \\
\text { Th a b a n e, } \\
\text { Foster y } \\
\text { Bédrad, } 2009\end{array}$ & $\begin{array}{l}283 \text { cuidadores } \\
\text { cónyuges de la } \\
\text { persona cuidada. }\end{array}$ & $\begin{array}{l}\text { - Índice de características } \\
\text { sociodemográficas } \\
\text { - Índice de Barthel } \\
\text { - Robinson Caregiver Social } \\
\text { Strain } \\
\text { - Utilización de los servicios }\end{array}$ & $\begin{array}{l}\text { Los participantes fueron } \\
\text { c a ptados por } \\
\text { enfermeras y otros } \\
\text { trabajadores. Se les } \\
\text { habló del estudio y se le } \\
\text { mandó una carta a su } \\
\text { domicilio. Si se } \\
\text { mostraban conformes } \\
\text { se iniciaban una serie } \\
\text { de entrevistas } \\
\text { telefónicas. }\end{array}$ & $\begin{array}{l}\text { - } \text { Género del cuidador } \\
\text { - } \quad \text { Edad del cuidador } \\
\text { - } \text { Nivel de estudios } \\
\text { - } \text { Percepción de la salud propia } \\
\text { - Horas semanales dedicadas al cuidado } \\
\text { - Semanas dedicadas a ser cuidador de } \\
\text { - } \quad \text { Torma activa } \\
\text { - Uso de los servicios }\end{array}$ \\
\hline $\begin{array}{l}\text { Jessup, Bakas, } \\
\text { McLennon y } \\
\text { Weaver, } 2015\end{array}$ & $\begin{array}{l}243 \text { cuidadores de } \\
\text { supervivientes de } \\
\text { infarto. }\end{array}$ & $\begin{array}{l}\text { - Test sobre datos demográficos } \\
\text { - Oberst caregiving burden } \\
\text { scale } \\
\text { Cuestionario sobre la salud } \\
\text { del paciente-9 (PHQ-9) } \\
\text { - Bakas Caregiving Outcomes } \\
\text { Scale (BCOS) } \\
\text { - Stroke Specific Quality of Life } \\
\text { Scale }\end{array}$ & $\begin{array}{l}\text { Mediante base de datos } \\
\text { de familias cuidadoras } \\
\text { en el medio oeste. Se } \\
\text { mandaron e-mails a las } \\
\text { familias para que dieran } \\
\text { su consentimiento antes } \\
\text { de llevar a cabo una } \\
\text { serie de Ilamadas } \\
\text { telefónicas. }\end{array}$ & $\begin{array}{l}\text { - } \text { Dificultad } \\
\text { - Síntomas depresivos } \\
\text { - } \text { Discapasiosidales de la persona cuidada } \\
\text { - } \text { Género del cuidador } \\
\text { - } \text { Raza del cuidador } \\
\text { - Relación coidador la persona cuidada }\end{array}$ \\
\hline
\end{tabular}

En el primer artículo, de Perrin et al, se trabajó con una muestra de 81 personas. Se utilizaron las siguientes escalas:

Información demográfica: Test realizado por los investigadores, incluyó los datos edad, género, estado sentimental, nivel de educación, situación laboral, horas semanales y tiempo total dedicado al cuidado, relación del cuidador y el paciente.

Cuestionario sobre la salud del paciente-9 (PHQ-9): este es un test au- toadministrado de 9 ítems de screening de síntomas depresivos utilizado en estudios clínicos y epidemiológicos. (7)

Escala de satisfacción con la vida (SWLS): este es un cuestionario autoadministrado de 5 ítems de medición de la satisfacción con la vida. La versión en español tiene propiedades psicométricas. (7)

Escala de autoestima de Rosemberg (RSES): cuestionario de 10 ítems acerca de la percepción de la propia valía. 
Inventario de ansiedad estadorasgo (STAI): es un cuestionario de 40 cuestiones y autoadministrado, que mide la ansiedad mediante dos subescalas. La subescala $S$ mide la intensidad de la ansiedad como un estado emocional y la subescala $\mathrm{T}$ mide la ansiedad como un rasgo de personalidad, distinto de la respuesta a una situación específica. (7)

Escala de Zarit: mide la carga de cuidado. Se completa de igual forma mediante una escala Likert del 0 al 4, donde 0 significa el nivel más bajo y el 5 el nivel más alto. Escalas más altas se asocian con mayor carga asociada al cuidado. (8)

Interpersonal Support Evaluation List (versión corta) (ISEL-12): es un instrumento que mide el apoyo social percibido por el paciente en varios ámbitos: sentimiento de pertenencia, aprecio y apoyo tangible. (7)

Cuestionario de salud (versión corta) (SF-36): se utilizó para medir la calidad de vida asociada a la salud. Mide ocho dominios que son función física, rol físico, dolor, funcionamiento social, rol emocional, salud mental y salud general. (7)

En este artículo los resultados se recogieron mediante una entrevista de 40 minutos con la persona cuidadora. En esta, se evaluaban varios factores que relacionaban género y cuidado. Las mu- jeres mostraron el doble de síntomas de depresión que los hombres $(p=0.00)$ y mayores niveles de ansiedad tanto como rango $(p=0.00)$. Los niveles de carga fueron más elevados en las mujeres, con menores niveles de vitalidad $(p=0.00)$. Así, también se encontraron niveles de salud más bajos en mujeres que en hombres $(p=0,00)$.

En el segundo artículo, se eligieron cuidadores que fueran pareja de la persona cuidada. Las escalas utilizadas fueron las siguientes:

- Índice de características sociodemográficas: realizado por los autores, se utilizó para medir variables como la edad del cuidador, género y tiempo dedicado al cuidado.

- Índice de Barthel: este test fue aplicado a la persona cuidada. Mide independencia funcional en el cuidado personal, movilidad y control de esfínteres, según la persona cuidadora. Los ítems se puntúan en función de si la persona puede realizar estas tareas por sí misma, con ayuda o es completamente dependiente. Para ello, se asignan puntuaciones que posteriormente se suman, pudiendo variar la puntuación final entre 0 y 20 , con un ni- 
vel más alto de autonomía cuanto más alta sea la puntuación.

- Robinson Caregiver Strain Scale: test de 13 ítems que mide la tensión asociada al cuidado. Se responde mediante preguntas de sí y no que se suman, dando un total de puntuación entre $0 \mathrm{y}$ 13 que indican mayor tensión a mayor puntuación.

- Utilización de los servicios: en este caso se llevó a cabo una lista con ayuda de las personas cuidadoras.

En esta muestra la mayor parte de las cuidadoras eran mujeres. No hubo diferencias estadísticamente significativas en ninguna otra característica demográfica, necesidades asistenciales en la vida diaria, ni horas dedicadas al cuidado. Sin embargo, hubo diferencias estadísticamente significativas en la tensión asociada al cuidado, siendo mucho mayor en mujeres que en hombres $(p=0.035)$.

En el último artículo se realizaron también entrevistas telefónicas con las familias cuidadoras, en este caso de pacientes supervivientes de infarto. Las escalas utilizadas fueron:
- Test sobre datos demográficos: Test ideado por los investigadores en el que preguntaban a la persona cuidadora acerca de cuestiones demográficas como edad, género, raza y tipo de relación con la persona cuidada.

- Oberst caregiving burden scale (OCBS). Consistente en una escala Likert 1-5 que los cuidadores deben rellenar asignando diferentes grados de dificultad a 15 ítems relacionados con aspectos del cuidado.

- Cuestionario sobre la salud del paciente-9 (PHQ-9).

- Bakas Caregiving Outcomes Scale (BCOS). En este cuestionario se pregunta de forma directa a la persona cuidadora acerca de los cambios que han tenido lugar en su vida y que están relacionados de forma directa con el cuidado que llevan a cabo. Cada una de las preguntas se responde con una puntuación entre 1 y 7 , donde 1 significa que ha cambiado hacia la peor situación posible y 7 es que ha cambiado hacia la mejor situación posible.

- Stroke Specific Quality of Life Scale: este test es realizado 
por la persona cuidadora, que nos da una clasificación de la discapacidad de la persona cuidada. Las subescalas incluidas en este cuestionario son la movilidad del paciente, autocuidado, pensamiento y personalidad. En una primera ronda se pide a las personas cuidadoras que elijan si la persona a la que cuidan podría hacer un número determinado de tareas, mediante una escala $\mathrm{Li}$ kert 1-5 donde 1 es no podría hacerlo en absoluto y 5 significa podría hacerlo sin ningún tipo de problema. En una segunda ronda se pregunta para la habilidad del paciente para realizar una serie de tareas. Mayores puntuaciones implican mayor grado de discapacidad.

En este estudio nuevamente la mayor parte de las cuidadoras eran mujeres. En cuanto a los síntomas depresivos asociados al cuidado, la relación de estos con el género $(p<0.001)$ y la raza fue significativa $(p=0.048)$. En cuanto a los síntomas depresivos, las mujeres también mostraron síntomas que se relacionaban de forma significativa con el género $(p<0.001)$. En cuanto a los cambios vitales derivados del cuidado, las mujeres y las personas no afroamerica- nas demostraron haber experimentado cambios vitales a peor $(p=0.008$ y $p=0.033)$.

En un estudio más a fondo, se encontró que las mujeres encontraban más dificultad que los hombres para encontrar a alguien que cuidara de la persona cuando ellas no podían llevar a cabo el cuidado $(p<0.001)$

En el primer artículo, de Perrin et al., se extraen como datos que las mujeres experimentan mayor depresión $(p=0.00)$ que los hombres, mayor ansiedad $(p=0.012)$, mayores niveles de carga $(p=0.14)$ y por tanto niveles de salud más bajos $(p=0.00)$.

Del artículo de Brazil, Thabane, Foster y Bédrad podemos extraer que la tensión asociada al cuidado fue mucho mayor en mujeres que en hombres $(p=0.035)$.

Por último, del artículo de Jessup, Bakas, McLennon y Weaver encontramos que la raza $(p=0.048)$ y el género $(p<0.001)$ están asociados de forma significativa con la depresión asociada al cuidado.

De estos artículos podemos extraer, pues, que las mujeres que cuidan (especialmente de raza no caucásica) experimentan mayores niveles de depresión, que las mujeres experimentan mayores niveles de ansiedad y tensión y 
que existen en ellas niveles de salud más bajos.

\section{Discusión}

La discusión, al igual que los resultados, los dividiremos en función de las diferentes mediciones en distintos tipos de cuidado. Nuestro objetivo principal era analizar la diferencia en la carga subjetiva asociada al cuidado, por lo que nos centraremos en debatir si existen diferencias estadísticamente significativas en cuanto a carga subjetiva relacionada con el género, lo que puede orientarnos hacia nuevas formas de entender el cuidado y la prevención hacia la persona cuidadora.

Carga asociada al cuidado en problemas de salud mental

En cuanto a los resultados de estos artículos, son comparables con otros estudios similares. Como ya hemos visto, el cuidado de familiares con alzheimer afecta a las personas que cuidan.

En concreto, las mujeres experimentan mayor carga y mayor estigma asociado al cuidado. Esto se corresponde con los resultados de otro estudio13, que nos muestra que las mujeres que cuidan a enfermeros de alzheimer sufren mayor número de enfermedades crónicas, asociadas a más carga asociada al cuidado y menor autoestima.
Carga asociada al cuidado en otras enfermedades

El artículo de Perrin et al. Afirma que las mujeres experimentan mayores niveles de carga asociada al cuidado, lo que se corresponde con otro artículo, que afirma que la carga subjetiva asociada al cuidado se relaciona con el sexo femenino de la persona cuidadora. (14)

Gracias a la actual comparación podemos constatar que existen diferencias estadísticamente significativas en la forma en que mujeres y hombres asumen el cuidado. Podemos afirmar que las mujeres, además de ser quienes asumen mayoritariamente el cuidado, también asumen mayor carga de cuidado, mayor carga física y mayor carga social. A esta carga se suma el estigma asociado al cuidado, mayor también en las mujeres y directamente relacionado con la carga.

La tensión asociada al cuidado también fue mayor en mujeres que en hombres, desembocando en niveles más altos de ansiedad y depresión. Esto se traduce en unos niveles de salud más bajos para el sector femenino de la población cuidadora.

Unos niveles más bajos de salud tienen como consecuencia mayores consultas a los centros de atención primaria, mayor sobrecarga asociada al cuidado y por tanto un cuidado de peor calidad tan- 
to para la persona cuidadora como para

la persona cuidada

\section{CONCLUSIONES}

Como podemos observar, sí que existen diferencias en la carga subjetiva asociada al cuidado, y ésta está claramente segmentada hacia el género femenino.

Como hemos comentado desde un primer momento, la mayor parte de las personas que cuidan son mujeres. Esto podría hacernos pensar que los mayores niveles de carga son simplemente una cuestión estadística. Es por ello que es importante realizar un análisis según datos más precisos y comparables, como es el estudio actual.

Para estudiar mejor estos fenómenos hacen falta nuevas investigaciones que en primer lugar creen instrumentos que faciliten la comparación y en segundo lugar utilicen estos instrumentos.

Desde las profesiones sociosanitarias debemos asumir un enfoque de género, partir de la base de que este es un problema que existe e investigar en la forma de paliarlo, para así poder asegurar una mayor calidad de vida a la población a la que atendemos. 


\section{BIBLIOGRAFÍA}

1. Comas D'Argemir D. Los cuidados de larga duración y el cuarto pilar del sistema de bienestar. Revista de Antropología Social. 2015;24(0):375-404.

2. Robinson C, Bottorff J, Pesut B, Oliffe J, Tomlinson $\mathrm{J}$. The Male Face of Caregiving. American Journal of Men's Health. 2014;8(5):409-426.

3. Casado-Mejía Ruiz-Arias E. Influence of Gender and Care Strategy in Family Caregivers' Strain: A Cross-Sectional Study. Journal of Nursing Scholarship. 2016;48(6):587-597.

4. Crespo López, M. Cuidadoras y cuidadores: el efecto del género en el cuidado no profesional de los mayores. Imserso, 2008.

5. Sánchez Herrera, B., Carrillo González, G. M., Barrera Ortiz, L., Chaparro Díaz, L. (2013). Carga del cuidado de la enfermedad crónica no transmisible. Aquichan. Vol. 13, No. 2, 247-260

6. Ocampo, JM, Herrera, JA, Torres, P, Rodríguez, JA, Loboa, L, García, CA. Sobrecarga asociada con el cuidado de ancianos dependientes. Colombia Médica [Internet]. 2007;38(1):40-46. Recuperado de: https://www.redalyc.org/articulo.oa?id=28338107

7. Perrin P, Panyavin I, Morlett Paredes A, Aguayo A, Macias M, Rabago B et al. A Disproportionate Burden of Care: Gender Differences in Mental Health, Health-Related Quality of Life, and Social Support in Mexican Multiple Sclerosis Caregivers. Behavioural Neurology. 2015;2015:1-9.

8. Kahn P, Wishart H, Randolph J, Santulli R. Caregiver Stigma and Burden in Memory Disorders: An Evaluation of the Effects of Caregiver Type and Gender. Current Gerontology and Geriatrics Research. 2016;2016:1-5.

9. Akpınar B, Küçükgüçlü Ö, Yener G. Effects of Gender on Burden Among Caregivers of Alzheimer's Patients. Journal of Nursing Scholarship. 2011;

10. Brazil K, Thabane L, Foster G, Bédard M. Gender differences among Canadian spousal caregivers at the end of life. Health \& Social Care in the Community. 2009;17(2):159-166.

11. Jessup N, Bakas T, McLennon S, Weaver M. Are there gender, racial or relationship differences in caregiver task difficulty, depressive symptoms and life changes among stroke family caregivers?. Brain Injury. 2014;29(1):17-24.

12. Creavin S, Wisniewski S, Noel-Storr A, Trevelyan C, Hampton T, Rayment D et al. Mini-Mental State Examination (MMSE) for the detection of dementia in clinically unevaluated people aged 65 and over in community and primary care populations. Cochrane Database of Systematic Reviews. 2016.

13. Chappell N, Dujela C, Smith A. Caregiver WellBeing. Research on Aging. 2014;37(6):623-645.
14. Casado-Mejía R, Ruiz-Arias E. Influence of Gender and Care Strategy in Family Caregivers' Strain: A Cross-Sectional Study. Journal of Nursing Scholarship. 2016;48(6):587-597. 